\title{
Long-Term Impact of Ivacaftor on Healthcare Resource Utilization Among People with Cystic Fibrosis in the United States
}

\author{
Teja Thorat · Lisa J. McGarry · Krutika Jariwala-Parikh • \\ Brendan Limone $\cdot$ Machaon Bonafede $\cdot$ Keval Chandarana • \\ Michael W. Konstan
}

Received: December 1, 2020 / Accepted: March 19, 2021 / Published online: April 28, 2021

(c) The Author(s) 2021

\section{ABSTRACT}

Introduction: Ivacaftor was first approved in 2012 for the treatment of a select population of individuals with cystic fibrosis (CF), a rare, lifeshortening genetic disease. Reductions in healthcare resource utilization (HCRU) associated with ivacaftor have been observed during limited follow-up and for selected outcomes in real-world studies. This study aimed to further describe the long-term impact of ivacaftor treatment on multiple measures of HCRU among people with $\mathrm{CF}$ (pwCF).

Methods: This retrospective study used US commercial and Medicaid claims data from 2011-2018. We included pwCF $\geq 6$ years of age with $\geq 1$ claim for ivacaftor and 12 months of continuous health plan enrollment before ivacaftor initiation ("pre-ivacaftor" period) who also had 36 months of continuous enrollment

T. Thorat $(\bowtie) \cdot$ L. J. McGarry · K. Chandarana Vertex Pharmaceuticals Incorporated, 50 Northern Ave, Boston, MA 02210, USA

e-mail: Teja_Thorat@vrtx.com

K. Jariwala-Parikh $\cdot$ B. Limone

IBM Watson Health, Boston, MA, USA

M. Bonafede

Veradigm Life Sciences, An Allscripts Healthcare

LLC, Chicago, IL, USA

M. W. Konstan

Case Western Reserve University and Rainbow

Babies and Children's Hospital, Cleveland, OH, USA and persistent ivacaftor use (i.e., no gap $\geq 90$ days between refills) following initiation ("post-ivacaftor" period). We compared comorbidities occurring pre-ivacaftor versus the last 12 months post-ivacaftor. HCRU outcomes included medication use, inpatient admissions, and outpatient office visits. We compared medication use pre-ivacaftor versus the last 12 months post-ivacaftor and inpatient admissions and outpatient office visits pre-ivacaftor versus the post-ivacaftor period annualized across 36 months.

Results: Seventy-nine pwCF met all criteria, including persistent ivacaftor use during the post-ivacaftor period. Ivacaftor treatment was associated with a significant reduction in pneumonia prevalence $(10.1 \%$ vs. $26.6 \%$; $p<0.001)$ and significantly fewer mean [SD] antibiotics claims (8.0 [7.3] vs. 12.3 [11.1]; $p<0.001)$ in the last 12 months post-ivacaftor versus pre-ivacaftor. In comparing the 36-month post-ivacaftor period to the pre-ivacaftor period, we also observed fewer mean [SD] annual inpatient admissions (0.2 [0.4] vs. 0.4 [0.7]), CF-related inpatient admissions (0.1 [0.2] vs. $0.2[0.5])$, and outpatient office visits (8.8 [4.9] vs. 9.9 [5.4]) (all, $p<0.05)$.

Conclusion: Long-term ivacaftor treatment reduced HCRU, consistent with trends observed in prior real-world studies. Our results support the sustained, long-term value of ivacaftor treatment in reducing CF burden. 
Keywords: Cystic fibrosis; Healthcare resource utilization; Ivacaftor; Real-world evidence

\section{Key Summary Points}

\section{Why carry out this study?}

Cystic fibrosis (CF) is a rare, lifeshortening, inherited disease associated with high use of healthcare resources, such as hospital visits, physician office visits, and prescription medications, throughout a person's life. In clinical trials and real-world studies, ivacaftor treatment has been shown to result in reductions in use of certain healthcare resources.

Given the progressive nature of $\mathrm{CF}$, it is important to understand the long-term impact of ivacaftor treatment on healthcare resource utilization in realworld settings and to continue to demonstrate its effectiveness to the CF community.

This study aimed to describe the long-term impact of ivacaftor treatment on use of healthcare resources among people with $\mathrm{CF}$ aged $\geq 6$ years.

\section{What was learned from the study?}

Using US administrative claims data, we showed that people with CF treated with ivacaftor for 36 months (3 years) had fewer all-cause and CF-related hospital stays, fewer physician visits, and lower prevalence of pneumonia, decreased use of mucolytics and opioids, and fewer prescription claims for antibiotics compared with before ivacaftor treatment.

These results support and reinforce the sustained, long-term value of ivacaftor treatment in lowering the burden of CF.

\section{DIGITAL FEATURES}

This article is published with digital features, including a summary slide, to facilitate understanding of the article. To view digital features for this article go to https://doi.org/10.6084/ m9.figshare.14236934.

\section{INTRODUCTION}

Cystic fibrosis (CF) is a progressive, life-shortening genetic disease caused by mutations in the cystic fibrosis transmembrane conductance regulator (CFTR) gene, resulting in absent or dysfunctional CFTR protein channels at the cell surface $[1,2]$. CF affects approximately 30,000 people in the United States and approximately 90,000 people globally $[3,4]$. CF is a multiorgan disease that leads to significant morbidity and mortality and requires complex polypharmacy and engagement with health systems from diagnosis (often at or shortly after birth) until death $[2,3,5,6]$. The progressive nature of the disease is evident in the observed annual decline of 1-3 percentage points in lung function among people with $\mathrm{CF}$ (pwCF), as well as their increasing healthcare burden over time [7-9]. Progressive loss of lung function is the leading cause of mortality $[2,10]$.

Ivacaftor is a small-molecule CFTR potentiator that increases CFTR channel opening probability, leading to increased chloride transport and thus addressing the underlying cause of the disease in pwCF with select mutations (e.g., gating mutations) [11]. Ivacaftor is approved in the USA, Europe, and other parts of the world for pwCF across a range of genotypes [12-14]. Indications vary among regions by age and CFTR mutations [12-14]. In clinical trials, ivacaftor use has demonstrated substantial reductions in pulmonary exacerbations (PEx), as well as improvement in lung function, reduction in annual rate of decline in lung function, and improvement in quality of life [15-17]. Since ivacaftor's approval in 2012, there has been growing interest in the long-term impact of ivacaftor on healthcare resource utilization (HCRU) in real-world settings. 
Administrative claims data are an important real-world data source for HCRU and are widely used to identify a sample of people who meet select criteria in order to evaluate the impact of a treatment on resource use [18]. Prior realworld studies that used administrative claims data to assess the impact of ivacaftor on HCRU have reported reductions in medication use, hospitalizations, and office visits over a followup period of 12 months after ivacaftor initiation $[19,20]$. To support and build on these findings, it is important to understand the long-term impact of ivacaftor treatment on HCRU given the progressive nature of the disease, as well as to continue demonstrating the effectiveness of ivacaftor to pwCF, physicians, and payers. Here, we evaluated the impact of long-term ivacaftor use on HCRU among pwCF $\geq 6$ years of age using administrative claims data in the USA.

\section{METHODS}

This non-interventional, retrospective study used data from the $\mathrm{IBM}^{\circledR}$ MarketScan ${ }^{\circledR}$ Commercial Claims and Encounters Database and the Medicaid Multi-State Database (both, IBM Watson Health; Sacramento, CA) spanning the period from January 1, 2011, to December 31, 2018. We identified pwCF with $\geq 1$ prescription claim for ivacaftor from January 1, 2012, through December 31, 2015. The date of the first prescription claim for ivacaftor was defined as the index date. PwCF were required to be $\geq 6$ years of age on the index date. A 12-month period of continuous enrollment in medical and pharmacy benefits (i.e., a health plan) prior to the index date, defined as the preivacaftor initiation period (pre-ivacaftor), was required for study inclusion. Individuals were required to have $\geq 1$ medical claim with an International Classification of Diseases (ICD)-9 (277.0x) or ICD-10 (E84.x) diagnosis code for CF during the pre-ivacaftor period or on the index date. PwCF were also required to have $\geq 36$ months of continuous enrollment in the health plan after the index date and persistent use of ivacaftor, defined as no gap of $\geq 90$ days after the end of supply and the start of the next refill. The 36-month period following ivacaftor initiation was defined as the post-ivacaftor initiation period (post-ivacaftor). The requirement for persistent ivacaftor use was important in order to evaluate the long-term impact of ivacaftor treatment with minimal or no treatment interruption.

Baseline characteristics, including age, sex, payer type, population density, insurance plan type, and year of ivacaftor initiation, were collected at the index date. Comorbidities were identified during the pre-ivacaftor period and during the last 12 months of the post-ivacaftor period (months 25-36) using ICD-9 or ICD-10 diagnosis codes in the primary or secondary position of inpatient and outpatient claims. Data on the following comorbidities were collected: anxiety, arthritis, asthma, bronchiectasis, chronic sinus disease, constipation, depression, diabetes, distal intestinal obstruction syndrome, gastroesophageal reflux disease (GERD), hemoptysis, insomnia, intestinal malabsorption, nasal polyps, osteopenia, osteoporosis, pancreatic insufficiency, pancreatitis, pneumonia, pulmonary infection, and pregnancy. HCRU outcomes included prescription medication use, all-cause and CF-related inpatient admissions, and outpatient office visits. Medication use was identified using National Drug Code and Healthcare Common Procedure Coding System codes. Data were collected on the use of the following medication classes: antibiotics (inhaled, oral, injectable, intravenous [IV], and other), bronchodilators, antidiabetic agents, antidepressants, antifungals, antihypertensives, anti-inflammatory agents, appetite stimulants, constipation treatments, digestive or pancreatic enzymes, immunosuppressants, mucolytics, opioids, ursodeoxycholic acid, and vitamins. CF-related admissions were defined by the ICD-9 or ICD-10 diagnosis code for $\mathrm{CF}$ in the primary diagnosis position on inpatient claims or in any position on outpatient claims.

Comorbidities and HCRU outcomes were summarized using descriptive statistics; continuous variables were summarized by mean (SD), and categorical variables, by frequency (\%). Comorbidities were calculated as number and proportion of pwCF with comorbidities during the pre-ivacaftor period and during the last 
12 months of the post-ivacaftor period. Prescription medication use was also reported as the number and proportion of pwCF with medication use and as the mean (SD) number of medication claims during the pre-ivacaftor period and the last 12 months of the post-ivacaftor period. All-cause and CF-related inpatient admissions and outpatient office visits were reported as the mean (SD) annual number of events during the pre-ivacaftor period and across the post-ivacaftor period (annualized). In addition, the numbers and proportions of pwCF with an all-cause inpatient and CF-related admission and outpatient office visit during the pre-ivacaftor period and the last 12 months of the post-ivacaftor period were reported. For comorbidities and HCRU outcomes, the absolute difference between the pre-ivacaftor period and the last 12 months of the post-ivacaftor period or the entire post-ivacaftor period was reported. Probability values for continuous variables were based on paired $t$ tests, and categorical variables were based on asymptotic McNemar tests. Values of $p<0.05$ were considered nominally significant; no multiplicity adjustment was conducted.

\section{Compliance with Ethics Guidelines}

This study did not involve primary data collection from human participants and relied on deidentified retrospective data from administrative claims; thus, institutional review board approval to conduct this study was not necessary. This study was conducted in accordance with Good Clinical Practice (GCP) as described in International Conference on Harmonisation (ICH) Guideline E6, GCP, Consolidated Guidance (April 1996). The ICH GCP guideline is consistent with the World Medical Assembly Declaration of Helsinki.

\section{RESULTS}

Of the total eligible pwCF with continuous health plan enrollment for 48 months during the pre- and post-ivacaftor periods $(n=121), 79$ pwCF were persistent in ivacaftor use during the entire 36-month post-ivacaftor period and were included in the analysis (Fig. 1). Most pwCF in this analysis were $<18$ years of age $(n=50$ $[63.3 \%]$ ), with the largest age group being those 6 to $<12$ years of age $(n=31 \quad[39.2 \%])$. The majority of pwCF $(64.6 \%)$ had commercial insurance. More than half of pwCF $(n=44$ [55.7\%]) initiated ivacaftor use in 2012, and the remaining $35 \quad(44.3 \%)$ initiated ivacaftor between 2013 and 2015 (Table 1). The prevalence of comorbidities in the pre-ivacaftor period is reported in Table 1.

In comparing the last 12 months of the postivacaftor period to the pre-ivacaftor period, no notable changes were observed in the prevalence of most frequently identified CF-related comorbidities occurring in $\geq 5 \%$ of pwCF, such as asthma, bronchiectasis, chronic sinus disease, diabetes, distal intestinal obstruction syndrome, GERD, intestinal malabsorption, nasal polyps, pancreatic insufficiency, and pulmonary infections (data not shown). However, a significant reduction in the prevalence of pneumonia was noted for individuals treated with ivacaftor, with a decrease in prevalence between the last 12 months of the post-ivacaftor period and the pre-ivacaftor period ( $10.1 \%$ vs. $26.6 \%$; absolute difference, -16.5 percentage points; $p<0.001$ ).

For HCRU outcomes, the proportion of pwCF receiving prescription medication decreased numerically for most drug classes after initiation of ivacaftor treatment (Fig. 2). No statistically significant changes were noted for most classes of prescription medication; however, significant reductions in the proportion of pwCF receiving inhaled and IV antibiotics, mucolytics, and opioids were observed (Fig. 2, Table 2). During the last 12 months of the post-ivacaftor period versus the pre-ivacaftor period, the proportion of pwCF receiving mucolytics decreased 11.4 percentage points (75.9\% vs. $87.3 \% ; p=0.003$ ) and receiving opioids decreased 12.7 percentage points (8.9\% vs. $21.5 \% ; p=0.012$ ). We also observed a numeric decrease in the proportion of pwCF receiving antibiotics and a significant reduction of $35 \%$ in the mean number of claims for antibiotics per person in the last 12 months of the post-ivacaftor period compared with the pre-ivacaftor period $(p<0.001)$ (Table 2$)$. Reductions were also observed in the mean number of antibiotics 


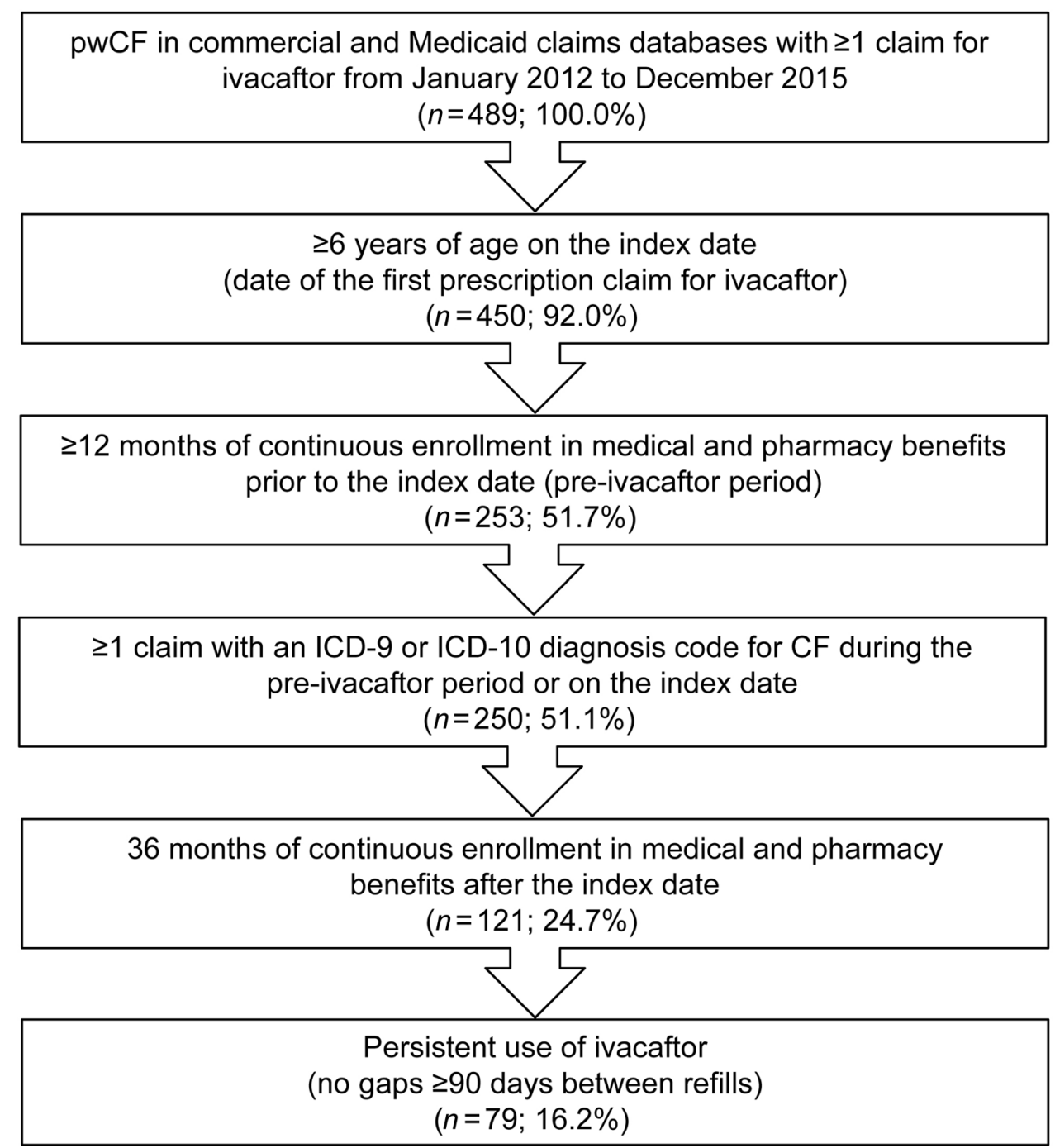

Fig. 1 Sample attrition. $C F$ cystic fibrosis, $I C D$ International Classification of Diseases, pwCF people with cystic fibrosis

claims per person by the route of administration during the last 12 months of the post-ivacaftor period compared with the pre-ivacaftor period (inhaled [26\% reduction; $p=0.012$ ], oral [26\% reduction; $p=0.003]$, injectable [70\% reduction; $p=0.043]$, and IV $[77 \%$ reduction; $p=0.028]$ ) (Table 2).

In addition, during the post-ivacaftor period, significant reductions were observed in inpatient admissions, CF-related inpatient admissions, and outpatient office visits (Table 3). The mean (SD) number of inpatient admissions and CF-related admissions per person was reduced by about half during the post-ivacaftor period compared with the pre-ivacaftor period $(0.2$
[0.4] vs. 0.4 [0.7]; $p=0.047 ; 0.1$ [0.2] vs. 0.2 $[0.5] ; p=0.024$, respectively). Also, we observed fewer outpatient office visits per person (8.8 [4.9] vs. 9.9 [5.4]; $p=0.022$ ) during the postivacaftor period compared with the pre-ivacaftor period. Furthermore, there was a 30\% reduction in the proportion of pwCF with an inpatient admission and 36\% reduction in the proportion of pwCF with a CF-related admission during the last 12 months of the post-ivacaftor period compared with the pre-ivacaftor period ( $17.7 \%$ vs. $25.3 \%$ and $8.9 \%$ vs. $13.9 \%$, respectively). Only a slight reduction was observed in the proportion of pwCF with outpatient office visits during the last 12 months of the post- 
Table 1 Demographics and comorbidities of pwCF

\begin{tabular}{|c|c|}
\hline & $\begin{array}{l}\text { pwCF } \\
(n=79)\end{array}$ \\
\hline Age, mean (SD), years & $19.5(15.6)$ \\
\hline \multicolumn{2}{|l|}{ Age categories, $n(\%)$} \\
\hline 6 to $<12$ years & $31(39.2)$ \\
\hline 12 to $<18$ years & $19(24.1)$ \\
\hline 18 to $<25$ years & $9(11.4)$ \\
\hline$\geq 25$ years & $20(25.3)$ \\
\hline Male, $n(\%)$ & $38(48.1)$ \\
\hline \multicolumn{2}{|l|}{ Payer, $n(\%)$} \\
\hline Commercial & $51(64.6)$ \\
\hline Medicaid & $28(35.4)$ \\
\hline \multicolumn{2}{|l|}{ Population density, $n$ (\%) } \\
\hline Urban & $57(72.2)$ \\
\hline Rural & $20(25.3)$ \\
\hline Unknown & $2(2.5)$ \\
\hline \multicolumn{2}{|l|}{ Insurance plan type, $n(\%)$} \\
\hline Comprehensive/indemnity & $12(15.2)$ \\
\hline $\mathrm{EPO} / \mathrm{PPO}$ & $33(41.8)$ \\
\hline POS/POS with capitation & $4(5.1)$ \\
\hline HMO & $25(31.6)$ \\
\hline CDHP/HDHP & $4(5.1)$ \\
\hline Unknown & $1(1.3)$ \\
\hline \multicolumn{2}{|l|}{ Index year, $n(\%)$} \\
\hline 2012 & $44(55.7)$ \\
\hline 2013 & $14(17.7)$ \\
\hline 2014 & $8(10.1)$ \\
\hline 2015 & $13(16.5)$ \\
\hline \multicolumn{2}{|l|}{$\begin{array}{l}\text { Comorbidities in } \geq 5 \% \text { of pwCF during } \\
\text { pre-ivacaftor period, } n(\%)\end{array}$} \\
\hline Distal intestinal obstruction syndrome & $23(29.1)$ \\
\hline Asthma & $27(34.2)$ \\
\hline Chronic sinus disease & $17(21.5)$ \\
\hline Pancreatic insufficiency ${ }^{a}$ & $60(75.9)$ \\
\hline
\end{tabular}

Table 1 continued

\begin{tabular}{lc}
\hline & $\begin{array}{l}\text { pwCF } \\
(\boldsymbol{n}=79)\end{array}$ \\
\hline Pneumonia & $21(26.6)$ \\
Pulmonary infection & $15(19.0)$ \\
Bronchiectasis & $13(16.5)$ \\
GERD & $10(12.7)$ \\
Constipation & $6(7.6)$ \\
Depression & $6(7.6)$ \\
Diabetes & $6(7.6)$ \\
Hemoptysis & $6(7.6)$ \\
Anxiety & $5(6.3)$ \\
Intestinal malabsorption & $4(5.1)$ \\
Nasal polyps & $4(5.1)$ \\
\hline
\end{tabular}

Demographics were measured on the index date. Comorbidities were measured in the 12 -month pre-ivacaftor period

$C D H P$ consumer-driven health plan, EPO exclusive provider organization, GERD gastroesophageal reflux disease, $H D H P$ high-deductible health plan, HMO health maintenance organization, ICD International Classification of Diseases, POS point of service, PPO preferred provider organization, $p w C F$ people with cystic fibrosis

a Calculated using ICD-9/10 diagnosis code for pancreatic insufficiency or the prescription medication use of digestive and pancreatic enzymes

ivacaftor period versus the pre-ivacaftor period (100\% vs. $98.7 \%)$.

\section{DISCUSSION}

This retrospective study of US commercial and Medicaid claims data showed that, over 36 months of ivacaftor treatment, substantial reductions in HCRU occurred, including a 50\% reduction in annual all-cause and CF-related inpatient admissions, compared with 12 months prior to ivacaftor initiation. Ivacaftor treatment was also associated with a 


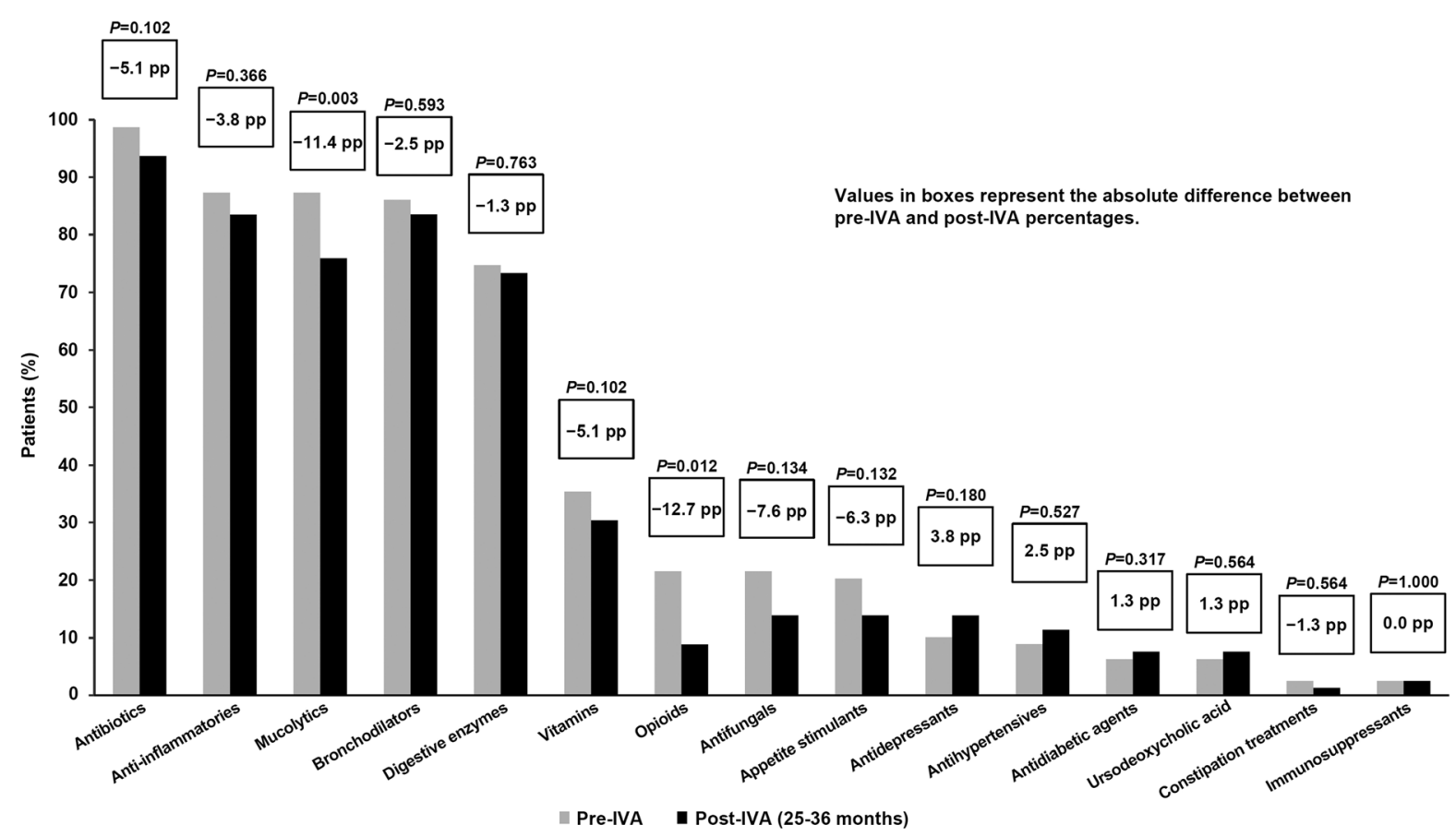

Fig. 2 Proportion of pwCF with $\geq 1$ claim for prescription medications during the pre-IVA period and the last 12 months of the 36-month post-IVA period. Diff difference, $p p$ percentage points, $p w C F$ people with cystic

significant reduction in outpatient office visits, a numeric reduction in use of several prescription medication classes, a significant reduction in the proportion of pwCF receiving either mucolytics or opioids, and a significant reduction in the number of antibiotics claims (inhaled, oral, injectable, and IV). We also observed a lower prevalence of pneumonia following 36 months of ivacaftor treatment than before ivacaftor initiation.

In a phase 3 clinical trial among participants $\geq 12$ years of age with $\geq 1$ G551D mutation, ivacaftor showed substantial improvements in lung function and body weight and a reduction in the risk of PEx events through 48 weeks, all of which were sustained for an additional 96 weeks in an open-label study, for a total study duration of approximately 33 months $[21,22]$. The current study showed a reduction in the use of antibiotics (typically administered to treat PEx events [23]) following ivacaftor treatment for as long as 36 months, suggesting a reduction in the number of PEx events in a real- fibrosis, post-IVA post-ivacaftor, pre-IVA pre-ivacaftor. Medication use was measured during the last 12 months of the post-IVA period. $p$ values are based on asymptotic McNemar tests

world setting. Additionally, this study demonstrated the longer-term impact of ivacaftor on comorbidities, inpatient admissions, and outpatient office visits in routine clinical practice.

The impact of ivacaftor on HCRU has been reported in several real-world studies, usually over a follow-up period of 12 months $[16,19,24-27]$. A published study using the same data sources as the current study found that, among commercially insured pwCF in the USA, use of ivacaftor for 12 months was associated with a 55\% reduction in the proportion of pwCF who had any inpatient admission, with a more pronounced reduction of $78 \%$ in the proportion of pwCF who had a CF-related inpatient admission [19]. Another study, which used data from the US Cystic Fibrosis Foundation Patient Registry to compare 12 months after ivacaftor initiation to 12 months before, reported a similar reduction of $65 \%$ in the rate of inpatient admissions [28]. Similarly, interim analyses of observational real-world studies across multiple centers in European countries 
Table 2 Proportion of pwCF and mean number of filled prescriptions for antibiotics during the pre-IVA period and the last 12 months of the 36 -month post-IVA period $(n=79)$

\begin{tabular}{|c|c|c|c|c|c|c|c|c|}
\hline & \multicolumn{4}{|c|}{ Proportion of pwCF with filled prescriptions } & \multicolumn{4}{|c|}{ Filled prescriptions } \\
\hline & $\begin{array}{l}\text { Pre- } \\
\text { IVA, } \\
n(\%)\end{array}$ & $\begin{array}{l}\text { Post- } \\
\text { IVA, } \\
n(\%)^{\mathrm{a}}\end{array}$ & $\begin{array}{l}\text { Absolute } \\
\text { difference, } \\
\text { (percentage } \\
\text { points) }\end{array}$ & $p$ value $\mathrm{e}^{\mathrm{b}}$ & $\begin{array}{l}\text { Pre-IVA, } \\
\text { mean } \\
(\mathrm{SD})\end{array}$ & $\begin{array}{l}\text { Post- } \\
\text { IVA, } \\
\text { mean } \\
(\mathrm{SD})^{\mathrm{a}}\end{array}$ & $\begin{array}{l}\text { Absolute } \\
\text { difference, } \\
\text { (percentage } \\
\text { points) }\end{array}$ & $p$ value $^{\mathrm{b}}$ \\
\hline $\begin{array}{l}\text { Antibiotics } \\
\text { (all) }\end{array}$ & $78(98.7)$ & $74(93.7)$ & -5.1 & 0.102 & $12.3(11.1)$ & $8.0(7.3)$ & -4.3 & $<0.001$ \\
\hline Inhaled & $42(53.2)$ & $30(38.0)$ & -15.2 & 0.007 & $2.2(2.9)$ & $1.5(2.5)$ & -0.6 & 0.012 \\
\hline Oral & $77(97.5)$ & $74(93.7)$ & -3.8 & 0.257 & $7.2(5.8)$ & $5.3(5.0)$ & -1.8 & 0.003 \\
\hline Injectable & $15(19.0)$ & $8(10.1)$ & -8.9 & 0.071 & $1.0(3.0)$ & $0.3(1.2)$ & -0.6 & 0.043 \\
\hline Intravenous & $19(24.1)$ & $9(11.4)$ & -12.7 & 0.012 & $1.3(4.8)$ & $0.3(1.0)$ & -1.1 & 0.028 \\
\hline Other & $15(19.0)$ & $12(15.2)$ & -3.8 & 0.513 & $0.4(1.0)$ & $0.3(0.9)$ & -0.1 & 0.644 \\
\hline
\end{tabular}

Post-IVA post-ivacaftor, pre-IVA pre-ivacaftor, $p w C F$ people with cystic fibrosis

${ }^{a}$ Medication use was measured during the last 12 months of the post-IVA period

${ }^{\mathrm{b}} p$ values are based on paired $t$ tests for continuous variables and asymptotic McNemar tests for categorical variables

that compared the 12 months after ivacaftor initiation to the 12 months prior to initiation have reported reductions in the rate of all-cause inpatient admissions ranging from $60-62 \%$, along with a $70-79 \%$ reduction in the rate of PEx requiring hospitalization [24-26]. Limited real-world studies have assessed the impact of ivacaftor on HCRU outcomes over a longer follow-up period. A study of 80 pwCF from the Irish CF registry reported a reduction of $38 \%$ in the use of oral and IV antibiotics; however, the study also reported a nonsignificant reduction of $18 \%$ in per-person inpatient admissions after 3 years of ivacaftor treatment compared with 1 year prior to ivacaftor initiation [29]. Additionally, the third interim analysis of a study assessing ivacaftor impact in pwCF in Europe reported decreases in all-cause inpatient admissions and acute antibiotic medications for up to 36 months of ivacaftor treatment compared with 12 months pre-ivacaftor initiation [27]. A post-authorization safety study using national CF patient registries in the USA and UK assessed the impact of ivacaftor over 5 years in the USA and 4 years in the UK. The study reported an approximately $40 \%$ lower risk of inpatient admissions along with improvement in clinical outcomes in an ivacaftor-treated cohort versus a matched-comparator cohort during the followup years [16]. Notably, results from the current study using US administrative claims data over a follow-up of 36 months after ivacaftor initiation reported reductions in inpatient admissions and antibiotics use that were highly consistent with results from other real-world studies; taken together, these findings further confirm the durability of ivacaftor impact over a longer follow-up period.

Reduction in HCRU is not only an important surrogate for clinical outcomes and a measure of health system burden, but also a means to quantify disease burden. The reductions in HCRU that we observed following ivacaftor initiation are likely to have a positive impact on both time devoted to managing $\mathrm{CF}$ and $\mathrm{CF}$ treatment burden. Previous studies have documented an increase in HCRU over time in pwCF, given the progressive nature of the disease $[7,8,30]$; however, we observed the opposite with ivacaftor therapy, namely, a reduction in HCRU following ivacaftor treatment for 36 months. These findings highlight the sustained benefit of ivacaftor treatment in 
Table 3 All-cause and CF-related inpatient admissions and outpatient office visits in pwCF during the pre-IVA period and the 36-month post-IVA period $(n=79)$

\begin{tabular}{llllll}
\hline & \multicolumn{2}{l}{ Events, mean $(\mathbf{S D}), \boldsymbol{n}^{\mathbf{a}}$} & & Absolute difference $^{\mathbf{b}}$ & $\boldsymbol{p}$ value $^{\mathbf{c}}$ \\
\cline { 2 - 3 } & Pre-IVA & Post-IVA & & \\
\hline All-cause inpatient admissions & $0.4(0.7)$ & $0.2(0.4)$ & 0.1 & 0.047 \\
CF-related inpatient admissions & $\mathrm{d}$ & $0.2(0.5)$ & $0.1(0.2)$ & 0.1 & 0.024 \\
Outpatient office visits & $9.9(5.4)$ & $8.8(4.9)$ & 1.1 & 0.022 \\
\hline
\end{tabular}

$C F$ cystic fibrosis, ICD International Classification of Diseases, post-IVA post-ivacaftor, pre-IVA pre-ivacaftor, pwCF people with cystic fibrosis

${ }^{a}$ The mean values for outcomes are reported as mean (SD) annual number of events during the 12-month pre-IVA period and as annualized values across the 36-month post-IVA period

${ }^{\mathrm{b}}$ Due to rounding, the numbers presented may not add up precisely

${ }^{c} p$ values are based on paired $t$ tests

d CF-related events were defined by a CF ICD-9 (277.0x) or ICD-10 (E84.x) in the primary diagnosis position on inpatient claims or any position on non-inpatient claims

terms of HCRU outcomes over multiple years of follow-up.

Our study has a number of limitations. The 72-month period from 2012 (when ivacaftor was approved) to 2018, which was our window for identifying pwCF who had 48 months of continuous health plan enrollment, was relatively short. Thus, all pwCF who initiated ivacaftor from 2016 to 2018 were excluded by default due to lack of follow-up. Therefore, our study sample included only the subset of pwCF who initiated ivacaftor between 2012 and 2015 and were persistent for 36 months, resulting in a small number of pwCF included in the final analysis. We identified the demographic characteristics of populations at different stages of patient attrition from Fig. 1 to compare pwCF with persistent ivacaftor use in the final sample $(n=79)$ with the following nested sample populations prior to implementation of exclusion criteria: (1) pwCF $\geq 6$ years of age on the index date $(n=450), \quad(2)$ pwCF $\geq 6$ years of age with $\geq 12$ months of continuous enrollment in a health plan prior to the index date $(n=253)$, and (3) pwCF $\geq 6$ years of age with $\geq 12$ months of continuous enrollment in a health plan prior to the index date and at $\geq 36$ months of continuous enrollment in a health plan postindex date $(n=121)$. The demographic characteristics such as sex, payer type (commercial vs. Medicaid), and population density (urban vs. rural) in the final sample were qualitatively similar to the abovementioned sample populations during patient attrition. The inclusion criterion of persistent ivacaftor use during 36 months of continuous enrollment in a health plan post-index date required for the final sample was associated with relatively younger pwCF, with a mean (SD) age of 19.5 (15.6) years compared with 22.3 (13.2) years and 22.0 (13.9) years for pwCF $\geq 6$ years of age on the index date and pwCF $\geq 6$ years of age with $\geq 12$ months of continuous enrollment in a health plan prior to the index date, respectively. Across the identified sample populations during patient attrition, pwCF initiating ivacaftor in 2012 constituted the largest proportion of the sample population. Future research to evaluate the long-term impact of ivacaftor with larger sample sizes should be considered. Furthermore, this analysis has inherent limitations due to its reliance on claims data, which are designed for claims processing and reimbursement, not for research purposes; thus, this analysis lacks information on clinical and laboratory test results, including genotyping and measures of lung function. Due to the lack of clinical and genotype information in the claims data, it is not possible to identify a matched, untreated comparator arm in order to control for change in outcomes over time due to natural disease progression. Hence, we used a study 
design that compared outcomes before and after the initiation of ivacaftor treatment. As a result, our comparison of the outcomes of pwCF who received ivacaftor over a long period versus their treatment burden before ivacaftor initiation may have underestimated the benefits of ivacaftor treatment. Future studies are warranted to evaluate the long-term impact of ivacaftor using untreated contemporaneous controls to effectively account for natural disease progression. Our analysis excluded pwCF who died prior to 36 months of continuous enrollment in a health plan post-index date. This could have possibly resulted in selection of an ivacaftortreated cohort with less severe disease characteristics; however, the study design of pre-/postivacaftor treatment allows each person with CF to serve as their own control, thus accounting for disease severity, and should not have biased the results. In this study, data for commercialand Medicaid-insured populations were pooled and presented together to maximize sample size; however, it has been shown that Medicaidinsured individuals tend to be sicker and have poor health outcomes; thus, the results reported here may not be fully representative of either population [31, 32]. Subgroup analyses by age were not reported due to the small sample size and unequal payer mix in the study population. The methodology did not include a multiplicity adjustment, and the $p$ values should therefore be considered nominal.

This study focused on pwCF $\geq 6$ years of age; future studies should focus on evaluating the long-term impact of ivacaftor in younger age groups in order to demonstrate ivacaftor's ability to modify disease progression and prevent irreversible organ damage [33, 34].

\section{CONCLUSION}

This analysis builds on a growing body of literature estimating the impact of ivacaftor in reducing HCRU among pwCF by evaluating ivacaftor use over a longer time period and multiple endpoints. Our results support and reinforce the sustained long-term value of treating pwCF with ivacaftor, as observed through impact on HCRU. These findings are consistent with those of previous clinical and real-world studies of ivacaftor conducted over short- and long-term follow-up periods from a range of data sources.

\section{ACKNOWLEDGEMENTS}

Funding. This study was supported by Vertex Pharmaceuticals Incorporated, Boston, MA, USA. All authors had full access to all data in this study and take complete responsibility for the integrity of the data and the accuracy of the data analysis. Vertex Pharmaceuticals Incorporated also funded the journal's Rapid Service Fee.

Authorship. All named authors meet the International Committee of Medical Journal Editors (ICMJE) criteria for authorship for this article, take responsibility for the integrity of the work as a whole, and have given their approval for this version to be published.

Medical writing, editorial, and other assistance. Editorial coordination and support were provided by Augustine Obi, PharmD, of Vertex Pharmaceuticals Incorporated; Augustine Obi may own stock or stock options in that company. Medical writing and editorial support were provided under the direction of the authors by JoAnna Anderson, PhD, CMPP. JoAnna Anderson is an employee of ArticulateScience LLC, which received funding from Vertex Pharmaceuticals Incorporated.

Disclosures. All authors received nonfinancial support (assistance with manuscript preparation) from ArticulateScience LLC, which received funding from Vertex Pharmaceuticals Incorporated. Additional disclosures are as follows: Teja Thorat, Lisa J. McGarry, and Keval Chandarana are employees of Vertex Pharmaceuticals Incorporated and may own stock or stock options in that company. Krutika Jariwala-Parikh and Brendan Limone are employees of IBM Watson Health Company, which received a research contract to conduct this study with and on behalf of Vertex 
Pharmaceuticals Incorporated. Machaon Bonafede is a former employee of IBM Watson Health Company, which received a research contract to conduct this study with and on behalf of Vertex Pharmaceuticals Incorporated. Michael W. Konstan discloses grants and personal fees from Anthera, AzurRx BioPharma, the Cystic Fibrosis Foundation, Laurent Pharmaceuticals, and Vertex Pharmaceuticals Incorporated; personal fees from Celtaxsys, Chiesi, Ionis Pharmaceuticals, Kala Pharmaceuticals, Merck, Paranta Biosciences, pH Pharma, and Santhera; and grants from the National Institutes of Health.

Compliance with ethics guidelines. This study did not involve primary data collection from human participants and relied on deidentified retrospective data from administrative claims; thus, institutional review board approval to conduct this study was not necessary.

Data availability. Data sharing is not applicable to this article because no data sets were generated or analyzed during the current study. Data used in this study were obtained from pre-existing data sets in the IBM $^{\circledR}$ MarketScan ${ }^{\circledR}$ Commercial Claims and Encounters Database and Medicaid Multi-State Database. Information on available resources and services related to these databases is available on the IBM MarketScan Research Database website (https://www.ibm.com/products/marketscanresearch-databases).

Open Access. This article is licensed under a Creative Commons Attribution-NonCommercial 4.0 International License, which permits any non-commercial use, sharing, adaptation, distribution and reproduction in any medium or format, as long as you give appropriate credit to the original author(s) and the source, provide a link to the Creative Commons licence, and indicate if changes were made. The images or other third party material in this article are included in the article's Creative Commons licence, unless indicated otherwise in a credit line to the material. If material is not included in the article's Creative
Commons licence and your intended use is not permitted by statutory regulation or exceeds the permitted use, you will need to obtain permission directly from the copyright holder. To view a copy of this licence, visit http:// creativecommons.org/licenses/by-nc/4.0/.

\section{REFERENCES}

1. Riordan JR, Rommens JM, Kerem B, Alon N, Rozmahel R, Grzelczak Z, et al. Identification of the cystic fibrosis gene: cloning and characterization of complementary DNA. Science. 1989;245(4922): 1066-73. https://doi.org/10.1126/science.2475911.

2. Ratjen F, Bell SC, Rowe SM, Goss CH, Quittner AL, Bush A. Cystic fibrosis. Nat Rev Dis Primers. 2015;1: 15010. https://doi.org/10.1038/nrdp.2015.10.

3. Cystic Fibrosis Foundation. Cystic Fibrosis Foundation Patient Registry: annual data report 2019. Bethesda: Cystic Fibrosis Foundation; 2020.

4. Bell SC, Mall MA, Gutierrez H, Macek M, Madge S, Davies JC, et al. The future of cystic fibrosis care: a global perspective. Lancet Respir Med. 2020;8(1): 65-124. https://doi.org/10.1016/S22132600(19)30337-6.

5. Schlüter DK, Griffiths R, Adam A, Akbari A, Heaven ML, Paranjothy S, et al. Impact of cystic fibrosis on birthweight: a population based study of children in Denmark and Wales. Thorax. 2019;74(5):447-54. https://doi.org/10.1136/thoraxjnl-2018-211706.

6. VanDevanter DR, Kahle JS, O'Sullivan AK, Sikirica S, Hodgkins PS. Cystic fibrosis in young children: a review of disease manifestation, progression, and response to early treatment. J Cyst Fibros. 2016;15(2):147-57. https://doi.org/10.1016/j.jcf. 2015.09.008.

7. Hassan M, Bonafede MM, Limone BL, Hodgkins P, Sawicki GS. The burden of cystic fibrosis in the Medicaid population. Clinicoecon Outcomes Res. 2018;10:423-31. https://doi.org/10.2147/ceor. S162021.

8. Liou TG, Elkin EP, Pasta DJ, Jacobs JR, Konstan MW, Morgan WJ, et al. Year-to-year changes in lung function in individuals with cystic fibrosis. J Cyst Fibros. 2010;9(4):250-6. https://doi.org/10.1016/j. jcf.2010.04.002.

9. Rubin JB, Limone B, Adolph N, Konstan M. Health care resource utilization among school-aged children with cystic fibrosis. J Manag Care Spec Pharm. 
2017;23(3-a Suppl):S49-50. https://doi.org/10. 18553/jmcp.2017.23.3-a.s1.

10. O'Sullivan BP, Freedman SD. Cystic fibrosis. Lancet. 2009;373(9678):1891-904. https://doi.org/10.1016/ S0140-6736(09)60327-5.

11. Yu H, Burton B, Huang C-J, Worley J, Cao D, Johnson JP Jr, et al. Ivacaftor potentiation of multiple CFTR channels with gating mutations. J Cyst Fibros. 2012;11(3):237-45. https://doi.org/10.1016/ j.jcf.2011.12.005.

12. Kalydeco (ivacaftor) [package insert]. Boston: Vertex Pharmaceuticals Incorporated; 2020. https://pi. vrtx.com/files/uspi_ivacaftor.pdf. Accessed 30 Nov 2020.

13. Kalydeco (ivacaftor) [summary of product characteristics]. Dublin: Vertex Pharmaceuticals (Ireland) Limited; 2020. https://www.ema.europa.eu/en/ documents/product-information/kalydeco-eparproduct-information_en.pdf. Accessed 30 Nov 2020.

14. Kalydeco (ivacaftor) [product information]. St Leonards, NSW, Australia: Vertex Pharmaceuticals (Australia) Pty Ltd; 2020. https://apps.medicines. org.au/files/vrpkalyd.pdf. Accessed 22 May 2020.

15. Bell SC, Mainz JG, MacGregor G, Madge S, Macey J, Fridman M, et al. Patient-reported outcomes in patients with cystic fibrosis with a G551D mutation on ivacaftor treatment: results from a cross-sectional study. BMC Pulm Med. 2019;19(1):146. https://doi.org/10.1186/s12890-019-0887-6.

16. Volkova N, Moy K, Evans J, Campbell D, Tian S, Simard C, et al. Disease progression in patients with cystic fibrosis treated with ivacaftor: data from national US and UK registries. J Cyst Fibros. 2020;19(1):68-79. https://doi.org/10.1016/j.jcf. 2019.05.015.

17. Sawicki GS, McKone EF, Pasta DJ, Millar SJ, Wagener JS, Johnson CA, et al. Sustained benefit from ivacaftor demonstrated by combining clinical trial and cystic fibrosis patient registry data. Am J Respir Crit Care Med. 2015;192(7):836-42. https://doi.org/ 10.1164/rccm.201503-05780C.

18. Cadarette SM, Wong L. An introduction to health care administrative data. Can J Hosp Pharm. 2015;68(3):232-7. https://doi.org/10.4212/cjhp. v68i3.1457.

19. Feng LB, Grosse SD, Green RF, Fink AK, Sawicki GS. Precision medicine in action: the impact of ivacaftor on cystic fibrosis-related hospitalizations. Health Aff. 2018;37(5):773-9. https://doi.org/10. 1377/hlthaff.2017.1554.
20. Hassan M, Bonafede MM, Limone BL, Hodgkins P, Suthoff ED, Sawicki G. 28 Reduction in pulmonary exacerbations (PEx) after initiation of ivacaftor: a retrospective cohort study among patients with cystic fibrosis (CF) treated in real-world settings. J Cyst Fibros. 2016;15:S58. https://doi.org/10.1016/ S1569-1993(16)30268-5.

21. McKone EF, Borowitz D, Drevinek P, Griese M, Konstan MW, Wainwright C, et al. Long-term safety and efficacy of ivacaftor in patients with cystic fibrosis who have the Gly551Asp-CFTR mutation: a phase 3, open-label extension study (PERSIST). Lancet Respir Med. 2014;2(11):902-10. https://doi.org/10.1016/s2213-2600(14)70218-8.

22. Ramsey BW, Davies J, McElvaney NG, Tullis E, Bell SC, Drevinek P, et al. A CFTR potentiator in patients with cystic fibrosis and the G551D mutation. N Engl J Med. 2011;365(18):1663-72. https://doi. org/10.1056/NEJMoa1105185.

23. West N, Flume P, Cystic Fibrosis Foundation. Pulmonary exacerbations clinical care guidelines. 2008. https://www.cff.org/Care/Clinical-CareGuidelines/Respiratory-Clinical-Care-Guidelines/ Pulmonary-Exacerbations-Clinical-CareGuidelines/. Accessed 30 Nov 2020.

24. Castellani C, Colombo C, van der Ent CK, Simmonds NJ, Kinnman N, Hassan M, et al. WS05.4 Clinical effectiveness results from the first interim analysis of the VOCAL study; an observational study of ivacaftor in patients with cystic fibrosis and selected non-G551D gating mutations. J Cyst Fibros. 2018;17:S10. https://doi.org/10.1016/S15691993(18)30146-2.

25. Hubert D, Fajac I, Munck A, Marguet C, Benichou J, Payen-Champenois C, et al. PND80-an observational study of ivacaftor in patients with cystic fibrosis in France: first interim analysis of health care resource utilization from the BRIO study. Value Health. 2018;21:S342. https://doi.org/10.1016/j. jval.2018.09.2046.

26. Hubert D, Fajac I, Munck A, Marguet C, Benichou J, Payen-Champenois $\mathrm{C}$, et al. Clinical effectiveness from the first interim analysis of the BRIO study: an observational study of cystic fibrosis patients treated with ivacaftor in France. Pediatr Pulmonol. 2018;53(S2):160 (Abstract 34).

27. van der Ent K, Simmonds NJ, Colombo C, DeSouza C, Kinnman N, Thorat T, et al. An observational study of ivacaftor in people with cystic fibrosis and selected non-G551D gating mutations: outcomes from the third interim analysis of the VOCAL study. Pediatr Pulmonol. 2020;55(S2):S220. https:// doi.org/10.1002/ppul.25089. 
28. Rowe SM, Heltshe SL, Gonska T, Donaldson SH, Borowitz D, Gelfond D, et al. Clinical mechanism of the cystic fibrosis transmembrane conductance regulator potentiator ivacaftor in G551D-mediated cystic fibrosis. Am J Respir Crit Care Med. 2014;190(2):175-84. https://doi.org/10.1164/rccm. 201404-0703OC.

29. Kirwan L, Fletcher G, Harrington M, Jeleniewska P, Zhou S, Casserly B, et al. Longitudinal trends in real-world outcomes after initiation of ivacaftor. A cohort study from the Cystic Fibrosis Registry of Ireland. Ann Am Thorac Soc. 2019;16(2):209-16. https://doi.org/10.1513/AnnalsATS.201802-149OC.

30. Konstan MW, Wagener JS, Vandevanter DR, Pasta DJ, Yegin A, Rasouliyan L, et al. Risk factors for rate of decline in FEV1 in adults with cystic fibrosis. J Cyst Fibros. 2012;11(5):405-11. https://doi.org/ 10.1016/j.jcf.2012.03.009.

31. Stephenson AL, Sykes J, Stanojevic S, Quon BS, Marshall BC, Petren K, et al. Survival comparison of patients with cystic fibrosis in Canada and the United States: a population-based cohort study.
Ann Intern Med. 2017;166(8):537-46. https://doi. org/10.7326/m16-0858.

32. Tumin D, Crowley EM, Li SS, Wooten W, Ren CL, Hayes D Jr. Patterns of health insurance coverage and lung disease progression in adolescents and young adults with cystic fibrosis. Ann Am Thorac Soc. 2021;18(2):290-9. https://doi.org/10.1513/ AnnalsATS.201911-839OC.

33. Davies J, Wang LT, Panorchan P, Campbell D, Tian $S$, Higgins $M$, et al. WS06-4 Ivacaftor (IVA) treatment in patients 6 to $<12$ months old with cystic fibrosis with a CFTR gating mutation: results of a 2-part, single-arm, phase 3 study. J Cyst Fibros. 2019;18:S11. https://doi.org/10.1016/S15691993(19)30151-1.

34. Rosenfeld M, Wainwright CE, Higgins M, Wang LT, McKee C, Campbell D, et al. Ivacaftor treatment of cystic fibrosis in children aged 12 to $<24$ months and with a CFTR gating mutation (ARRIVAL): a phase 3 single-arm study. Lancet Respir Med. 2018;6(7):545-53. https://doi.org/10.1016/s22132600(18)30202-9. 